\title{
Distributed, Limbic Gray Matter Atrophy in Patients after Bacterial Meningitis
}

\author{
N.K. Focke, K. Kallenberg, A. Mohr, M. Djukic, R. Nau, and H. Schmidt
}

\begin{abstract}
BACKGROUND AND PURPOSE: The structural basis of cognitive sequelae after bacterial meningitis in humans is still poorly understood. In animal models and human autopsy cases, neuronal apoptosis of the hippocampal formation in particular seems to play an important role. Here, we aimed to analyze if BM entails MR imaging structural consequences in humans in vivo.
\end{abstract}

MATERIALS AND METHODS: We applied voxel-based morphometry in a cohort of BM survivors with normal conventional MR imaging after resolution of the acute inflammation to assess morphologic differences.

RESULTS: We found clear gray matter volume loss in the limbic system including the hippocampal formation, thalamus, and cingulate gyri bilaterally as well as in the temporal lobe. These results were corroborated by an alternative atlas-based method.

CONCLUSIONS: Even in patients with normal routine MR imaging results, clear-cut gray matter atrophy with a mesial temporal/limbic pattern was evident. The anatomic distribution is compatible with the neuropsychological deficit commonly observed in patients after BM. The similarity of the observed atrophy may point to causal link between BM and mesial temporal epilepsy.

ABBREVIATIONS: $B M=$ bacterial meningitis; $m$ TLE = mesial temporal lobe epilepsy; TIV = total intracranial volume; VBM = voxel-based morphometry

B acterial meningitis is associated with disabling sequelae in a significant proportion of patients. ${ }^{1}$ However, the structural basis of these in many cases is not clear. In a previous study, we found a normal conventional MR imaging result in 46 of 58 patients. The available evidence points toward an involvement of the hippocampal formation: an increased apoptosis in the dentate

Received May 8, 2012; accepted after revision July 27.

From the Departments of Clinical Neurophysiology (N.K.F.); Neurology (H.S.); Neuropathology (R.N.); Medical Psychology and Medical Sociology (H.S.); and Neuroradiology (K.K., A.M.), University of Göttingen, Göttingen, Germany; and Department of Geriatrics, Evangelisches Hospital, Göttingen (M.D., R.N.), Göttingen, Germany.

This study was partly supported by the DFG.

Author contributions: N.K.F. has conducted the VBM analysis and has written the first draft of the manuscript. K.K. was involved in the acquisition of the MR imaging scans and contributed to the writing of the manuscript. A.M. supervised the acquisition of the MR imaging scans and contributed to the writing of the manuscript. M.D. was involved in the conception of the study, recruitment/identification of study participants, and contribution to the writing of the manuscript. R.N. was involved in the conception of the study and contributed to the writing of the manuscript. H.S. was involved in the conception of the study, recruitment/identification of study participants, and data management, and has contributed to the writing of the manuscript.

Please address correspondence to Niels K. Focke, Department of Clinical Neurophysiology, University of Göttingen, Robert-Koch-Str 40, Göttingen, 37099, Germany; e-mail: nfocke@uni-goettingen.de

- Indicates open access to non-subscribers at www.ajnr.org

三 Indicates article with supplemental on-line tables

http://dx.doi.org/10.3174/ajnr.A3351 gyrus has been found after BM at autopsy. ${ }^{2}$ Another study ${ }^{3}$ showed bilateral hippocampal volume loss in patients with temporal lobe epilepsy with prior encephalitis or meningitis. In addition to the meningitis itself, the bacteriemia seems to play an important role in the development of apoptosis in bacterial meningitis, as demonstrated in a rat model of bacterial meningitis. ${ }^{4}$ Moreover, in another autoptic series, patients after bacterial meningitis displayed focal and diffuse axonal damage that was most prominent in the basal ganglia and in the hippocampal formation. ${ }^{5}$ In contrast to the sparse and often casuistic knowledge in humans, experimental murine, rat, and lapine BM models have extensively shown the important role of damage to the hippocampal formation mainly caused by apoptotic neuronal decay ${ }^{6,7}$ and to the neurons in the neocortex, mostly caused by necrosis. ${ }^{8,9}$

In our current study, we applied voxel-based morphometry, an unbiased MR imaging technique, to assess volumetric differences in patients after BM with normal conventional MR imaging results compared with healthy control participants; as an alternative approach, we confirmed the results with an atlas-based method. ${ }^{10}$

\section{MATERIALS AND METHODS Patients}

From the 58 patients of our previous, retrospective cohort study ${ }^{11}$ who had undergone a volumetric MR imaging scan, we excluded 


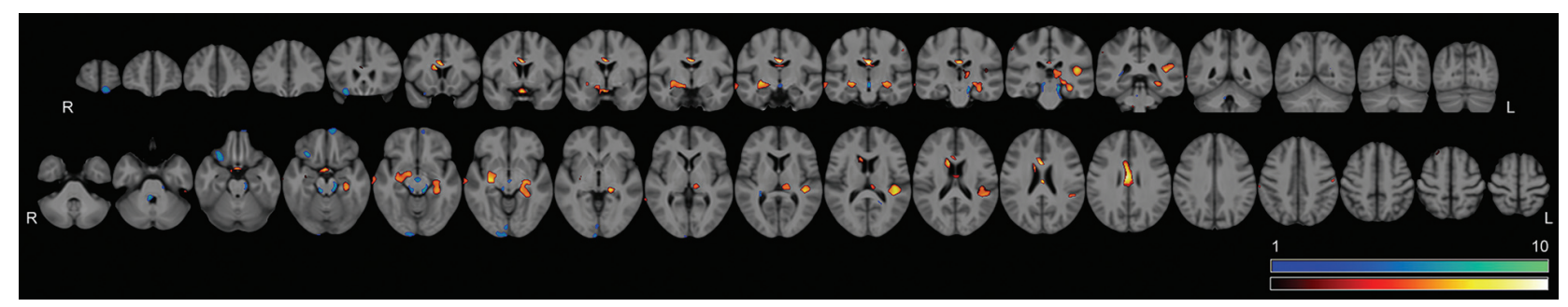

FIGURE. Group-level differences in SPM8. Significant volumetric differences $(P<.05$ family-wise error-corrected, peak level) between patients and healthy control participants are shown overlaid on the averaged, normalized T1-weighted image of the entire cohort. The color scale represents $t$ values: red-yellow colors are decreases (patients $<$ control participants), and blue-green colors are increases (patients $>$ control participants). Top row, Coronal sections (anterior to posterior). Bottom row, Axial sections (inferior to superior). Images are in radiologic convention (left in the image is right in the participants).

those with visible morphologic alterations (12/58). In 5 patients the original DICOM datasets were not available or had quality problems (eg, movement artifacts). Thus, 41 patients after BM with normal routine MR imaging results (as rated by board-certified neuroradiologists, who also rated a T2-fluid-attenuated inversion recovery sequence) were available for the further analysis and were compared with a control group of 12 healthy participants without a medical history of neurologic disease and with normal neurologic examination results. The mean age was $41.8 \pm$ 14.3 years (age range, 19-68 years) for patients and $49.2 \pm 10.5$ years (age range, 33-66 years) for control participants. A total of 18 patients $(43.9 \%)$ and 5 control participants $(41.7 \%)$ were women. Detailed biometric data of the enrolled participants are shown in On-line Table 1. All participants gave written informed consent, and the study was approved by the local ethics committee. The detailed inclusion and exclusion criteria are given in our previous publication. ${ }^{11}$

In brief, we included patients with a history of definite BM as evidenced by positive results on CSF culture or Gram stain or at least 2 distinctive signs of central nervous system infection (CSF leukocyte count $\geq 1000 /[$ micro]L, CSF lactate $\geq 3.0 \mathrm{mmol} / \mathrm{L}$, and CSF protein concentration $\geq 1000 \mathrm{mg} / \mathrm{L}$ ) plus clinical signs of meningitis. Exclusion criteria were age younger than 15 years or older than 70 years, a history of alcoholism or any other addiction disorder, any neurologic or psychiatric comorbidity possibly affecting the central nervous system, or known neoplastic diseases. Patients with BM from tuberculosis or borreliosis were also excluded. Thus, the spectrum of bacterial species included streptococcus pneumonia (27\%), Neisseria meningitides (27\%), Staphylococcus aureus (2\%), other streptococci (8\%), and Listeria monocytogenes (5\%). In $31 \%$ of patients, no identification was possible because of negative culture or Gram stain results.

\section{MR Imaging}

High-resolution T1-weighted 3D $(1.3 \times 1 \times 1 \mathrm{~mm})$ scans were acquired on a $1.5 \mathrm{~T}$ scanner (Philips, Best, the Netherlands) (3DT1-fast-field-echo: TE, $6.0 \mathrm{~ms}$; TR, $24.05 \mathrm{~ms}$; flip angle, 30 ; matrix, $130 \times 256 \times 256$ ).

\section{Voxel-Based Morphometry}

The original DICOM images were converted to the Neuroimaging Informatics Technology Initiative format and were analyzed off-line with statistical parametric mapping software, v8
(SPM8; http://www.fil.ion.ucl.ac.uk/spm/) running in Matlab 7.7 (MathWorks, Natick, Massachusetts). Images were segmented into tissue classes (gray matter, white matter, CSF, and nonbrain classes) by use of the revised unified segmentation model of SPM8 ("new segment") with default settings. Gray matter maps were normalized to Montreal Neurological Institute space in $1.5-\mathrm{mm}$ cubic resolution by use of the DARTEL (Diffeomorphic Anatomical Registration using Exponentiated Lie Algebra) procedure of SPM8. ${ }^{12}$ The resulting maps were modulated with the Jacobian determinants to preserve the local and global gray matter amounts and were convolved with an $8 \times 8 \times 8$-mm Gaussian kernel. We estimated the total intracranial volume by summing up the probability maps of gray matter, white matter, and CSF multiplied by voxel size (in native space) using a custom Matlab script. We then analyzed group differences of patients with BM compared with control participants by using the general linear model of SPM8 and a 2-group $t$ test including TIV, age, and sex as confounders of no interest. Differences between the patient and control groups with a threshold of $P<.05$ (corrected for multiple comparison with a family-wise error rate) were considered significant. Resulting statistical maps were overlaid on the averaged, normalized T1-weighted scan of the complete group with MRIcro (http://www.sph.sc.edu/comd/rorden/mricro.html). The FSL4.1 (FMRIB Software Library, http://www.fmrib.ox.ac.uk/fsl/) atlas tool (Harvard-Oxford cortical and subcortical atlases) was used to assess the anatomic location of the detected clusters (reporting only the highest-probability gray matter structure).

\section{Atlas-Based Volumetry}

In addition to the VBM analysis, we conducted an atlas-based volumetry. To this end, the modulated, normalized gray matter maps (not smoothed) were, in turn, masked with 83 anatomic labels of a maximal probability atlas based on 30 participants (n30r83 atlas). ${ }^{10}$ For further analysis, CSF labels were not considered, which left 78 distinct brain regions. The modulated gray matter voxels within each region were summed up and multiplied with the voxel volume with use of a Matlab script, resulting in one absolute gray matter volume per region. To account for global effects (eg, head size), we divided this regional volume by TIV (see above), yielding a dimensionless fractional value that was expressed in per million $(\%)$.

For each region, we analyzed differences between patients and control participants by using PASW Statistics v18 (IBM, Somers, 
New York) and a general linear model controlling for age and sex as confounders of no interest. Resulting $P$ values were corrected with Bonferroni error correction; a corrected $P<.05$ value was considered statistically significant.

\section{RESULTS}

\section{Voxel-Based Analysis}

VBM revealed significant loss of gray matter in the limbic system and in the temporal lobe (Figure). In particular, the hippocampal formations, the amygdalae, middle temporal gyri, left inferior temporal gyrus, and left planum temporale showed reduced gray matter volumes. Outside of the temporal lobe the left thalamus, the anterior cingulate gyrus, and the area of the mammillary bodies were affected. Gray matter increases compared with control participants were found around the brain stem, in the orbitofrontal area, and the occipital lobe. A detailed description of the detected clusters with their anatomic positions is shown in On-line Table 2.

\section{Atlas-Based Analysis}

When quantified with the alternative atlas-based method, the findings of the VBM analysis were generally confirmed (compare On-line Table 3). Significantly smaller gray matter volumes were found for the hippocampal formations, the left medial and inferior temporal gyri, the right cingulate gyrus (posterior part), the right parietal lobe, the left superior frontal gyrus, and the callosal area. Significantly increased gray matter volumes were detected in the brain stem, the left occipital lobe, both middle frontal gyri, the left parietal lobe, both anterior orbital gyri, the right superior frontal gyrus, the right superior parietal gyrus, both lateral orbital gyri, and the left posterior orbital gyrus.

\section{DISCUSSION}

In this analysis, we show that even in patients with normal routine MR imaging results, structural sequelae after BM are present. These encompass several brain regions-in particular, the temporal lobe and the limbic system. Our study was performed in patients without any visible substance deficits such as postvasospastic brain infarctions, lesions attributed to abscess formation/ evacuation, or other complications that BM can induce. In addition, care was taken during the recruitment to exclude any comorbidities, such as alcoholism, that could predispose to BM as well as directly causing atrophy. We hypothesize that the mesial temporal region has a specific susceptibility for injury caused by BM. Such predispositions have been described in BM in the hippocampal region in rabbits ${ }^{7,9}$ and in rats. ${ }^{13}$ Also, apoptotic lesions along the convexities of the brain have been found in rats. ${ }^{14} \mathrm{~A}$ histopathologic postmortem analysis of patients who died of bacterial meningitis also suggested a vulnerability of the mesial temporal region in humans. ${ }^{2}$ Because of the technical design, our findings are examiner-independent and did not only confirm the early region of interest-based hippocampal volume loss in a subgroup of patients with $\mathrm{BM}^{3}$ but also showed distributed atrophy. It is likely that the involvement of the limbic system (anterior cingulate gyrus, thalamus, mammillary bodies) reflects network effects. However, it remains uncertain if a single region, possibly the hippocampal formation, is the essential focus of pathophysi- ologic changes as suggested by animal studies or if several, or all, regions are affected in parallel. From a neuropsychological point of view, the hippocampal predominance of volume loss might explain a considerable part of the memory dysfunctions that survivors of BM often experience. ${ }^{11,15,16}$ In our previous study in the same cohort, ${ }^{11}$ we found significantly worse neuropsychological performance in BM for working memory, executive, and visoconstructive functions.

It is interesting to note that a striking similarity exists in the atrophy pattern in patients after BM compared with patients with mesial temporal lobe epilepsy without a history of bacterial meningitis. ${ }^{17,18}$ This atrophy pattern might represent an important morphologic factor for epilepsy after meningitis or encephalitis occurring in approximately $4 \%-5 \%$ of patients. This finding is in line with the observation that priming events, like febrile convulsions but also similar to meningitis or encephalitis, are frequently found in mesial temporal lobe epilepsy with hippocampal sclerosis but are less common in other types of epilepsy. ${ }^{19}$ However, other studies ${ }^{20,21}$ suggest that only meningitis before age 4 years is an important factor for the development of mTLE. Our sample, in contrast, was adult-onset BM; clinically overt epilepsy was found in only 1 patient during the observation period. Nevertheless, our findings may trigger further studies looking into the link between priming events and the development of epilepsy. The role of inflammatory mediators in epileptogenesis has received increasing attention lately. ${ }^{22}$

VBM also detected relative increases in gray matter volume in patients compared with control participants. Most of these increases occurred superficially in the pial or subpial area. A possible explanation for these findings is residual focal thickening of the meninges after BM or small subpial lesions that escaped visual detection by the radiologists. In particular, this effect is plausible for the brain stem and the subpial and ventricular regions.

\section{Study Limitations}

The present cohort of 41 consecutive patients was of moderate size for a VBM study. Thus, we could not infer that regions not showing significant differences were unaffected. To control the problem of multiple comparisons, we applied strict error correction both for the VBM as well as for the atlas-based analysis. The group size also did not allow a valid subgroup analysis concerning the bacterial species. Also, the control group of 12 patients was relatively small. However, the observed volumetric loss was consistent between the VBM and atlas-based method and was compatible with previous findings in animal models. ${ }^{7,9,13}$ Unfortunately, because of a replacement of our MR imaging scanner, the cohort could not be increased further because pooling of data between different MR imaging scanners for VBM analysis is challenging and can result in significant bias. ${ }^{23}$

\section{CONCLUSIONS}

$\mathrm{BM}$ survivors with a normal conventional MR imaging result show distinct gray matter atrophy in the limbic areas, with a focus on the mesial temporal region. This volume reduction is in accordance with histopathologic evidence in BM models and human autopsy cases. Moreover, the similarity of the observed atrophy 
may point to a causal link between BM and mesial temporal epilepsy.

ICMJE disclosures: Niels Focke-UNRELATED: Grants/Grants Pending: Jackstädt Foundation Research Grant; Payment for Lectures (including service on speaker bureaus): speaker honoraria from GlaxoSmithKline; Travel/Accommodations/ Meetings Expenses Unrelated to Activities Listed: UCB. Kai Kallenberg-RELATED: Grant: Volkswagen Stiftung; UNRELATED: Payment for Lectures (including service on speaker bureaus): Acandis, Pforzheim/Germany, Comments: speaker bureau (stroke/thrombectomy). Roland Nau-UNRELATED: Payment for Lectures (including service on speaker bureaus): Pfizer, Comments: lectures on "Treatment of Pain" (€500); Stock/Stock Options: Small amounts of stocks of pharmaceutical companies not involved in this project. Holger Schmidt-RELATED: Grant: Deutsche Forschungs Gemeinsachft grant; UNRELATED: Payment for Lectures (including service on speaker bureaus): speaker honorarium, (all €1000), Comments: the participants of all of these lectures were not involved in the current study; Stock/Stock Options: Bayer, UCB, Glaxo-Wellcome stocks; Travel/Accommodations/Meeting Expenses Unrelated to Activities Listed: Travel grants all lower than $€ 1000$ by Allergan, Merck, and Ipsen Pharma.

\section{REFERENCES}

1. Edmond K, Clark A, Korczak VS, et al. Global and regional risk of disabling sequelae from bacterial meningitis: a systematic review and meta-analysis. Lancet Infect Dis 2010;10:317-28

2. Nau R, Soto A, Bruck W. Apoptosis of neurons in the dentate gyrus in humans suffering from bacterial meningitis. J Neuropathol Exp Neurol 1999;58:265-74

3. Free SL, Li LM, Fish DR, et al. Bilateral hippocampal volume loss in patients with a history of encephalitis or meningitis. Epilepsia 1996;37:400-05

4. Østergaard C, Leib S, Rowland I, et al. Bacteremia causes hippocampal apoptosis in experimental pneumococcal meningitis. BMC Infect Dis 2010;10:1

5. Gerber J, Seitz RC, Bunkowski S, et al. Evidence for frequent focal and diffuse acute axonal injury in human bacterial meningitis. Clin Neuropathol 2009;28:33-39

6. Stringaris AK, Geisenhainer J, Bergmann F, et al. Neurotoxicity of pneumolysin, a major pneumococcal virulence factor, involves calcium influx and depends on activation of p38 mitogen-activated protein kinase. Neurobiol Dis 2002;11:355-68

7. Zysk G, Brück W, Gerber J, et al. Anti-inflammatory treatment influences neuronal apoptotic cell death in the dentate gyrus in experimental pneumococcal meningitis. J Neuropathol Exp Neurol 1996;55:722-28

8. Grandgirard D, Steiner O, Täuber MG, et al. An infant mouse model of brain damage in pneumococcal meningitis. Acta Neuropathol 2007;114:609-17

9. Leib SL, Kim YS, Chow LL, et al. Reactive oxygen intermediates contribute to necrotic and apoptotic neuronal injury in an infant rat model of bacterial meningitis due to group B streptococci. J Clin Invest 1996;98:2632-39

10. Hammers A, Allom R, Koepp MJ, et al. Three-dimensional maximum probability atlas of the human brain, with particular reference to the temporal lobe. Hum Brain Mapp 2003;19:224-47

11. Schmidt H, Heimann B, Djukic M, et al. Neuropsychological sequelae of bacterial and viral meningitis. Brain 2006;129:333-45

12. Ashburner J. A fast diffeomorphic image registration algorithm. Neuroimage 2007;38:95-113

13. Sury MD, Agarinis C, Widmer HR, et al. JNK is activated but does not mediate hippocampal neuronal apoptosis in experimental neonatal pneumococcal meningitis. Neurobiol Dis 2008;32:142-50

14. Blaser C, Wittwer M, Grandgirard D, et al. Adjunctive dexamethasone affects the expression of genes related to inflammation, neurogenesis and apoptosis in infant rat pneumococcal meningitis. PLoS One 2011;6:e17840

15. Weisfelt M, van de Beek D, Hoogman M, et al. Cognitive outcome in adults with moderate disability after pneumococcal meningitis. J Infect 2006;52:433-39

16. Merkelbach S, Sittinger H, Schweizer I, et al. Cognitive outcome after bacterial meningitis. Acta Neurol Scand 2000;102:118-23

17. Focke NK, Yogarajah M, Bonelli SB, et al. Voxel-based diffusion tensor imaging in patients with mesial temporal lobe epilepsy and hippocampal sclerosis. Neuroimage 2008;40:728-37

18. Keller SS, Roberts N. Voxel-based morphometry of temporal lobe epilepsy: an introduction and review of the literature. Epilepsia 2008;49:741-57

19. Mathern GW, Babb TL, Vickrey BG, et al. The clinical-pathogenic mechanisms of hippocampal neuron loss and surgical outcomes in temporal lobe epilepsy. Brain 1995;118:105-18

20. Marks DA, Kim J, Spencer DD, et al. Characteristics of intractable seizures following meningitis and encephalitis. Neurology 1992;42: 1513-18

21. O'Brien TJ, Moses H, Cambier D, et al. Age of meningitis or encephalitis is independently predictive of outcome from anterior temporal lobectomy. Neurology 2002;58:104-09

22. Friedman A, Dingledine R. Molecular cascades that mediate the influence of inflammation on epilepsy. Epilepsia 2011;52:33-39

23. Focke NK, Helms G, Kaspar S, et al. Multi-site voxel-based morphometry-not quite there yet. Neuroimage 2011;56:1164-70 